\title{
Vanishing condensates and anomalously light Goldstone modes in medium
}

\author{
Thomas D. Cohen \\ Department of Physics and Astronomy, University of Maryland, College Park, MD 20742, USA \\ Wojciech Broniowski \\ H. Niewodniczański Institute of Nuclear Physics, PL-31342 Cracow, POLAND
}

\begin{abstract}
We show that in a uniform medium, the vanishing of a particular condensate along with spontaneously broken symmetry imply the existence of an anomalously light pseudo-Goldstone mode. The consequences for a vanishing chiral condensate in nuclear matter are discussed.
\end{abstract}


Recently, Ericson [1] observed that if the chiral condensate, $\langle\bar{q} q\rangle$, goes to zero in nuclear matter at some density, one does not know a priori that chiral restoration occurred. The possibility exists that $\langle\bar{q} q\rangle$ can vanish without chiral restoration. In this letter, we show from very general arguments that if Ericson's scenario of a vanishing condensate without symmetry restoration is realized, then the pion's mass must be anomalously light in a sense which will be precisely defined.

The letter is organized as follows: We begin by introducing the problem in the very general context of the ground state (subject to a spatially uniform constraint) of an arbitrary quantum field theory with a nearly conserved current. Next we note the connection of this general problem to the problem of nuclear matter with a vanishing chiral condensate. We proceed to demonstrate for the general case that a vanishing condensate without symmetry restoration must imply an anomalously light pseudo-Goldstone boson. Finally, we discuss the implications of this result in the context of nuclear matter and simple pion condensation models and show why our results do not apply for the kaon condensation problem. We also briefly address the role of spatial correlations in $\bar{q} q$ in this problem. Before proceeding we wish to remind the reader that all of the results in this paper apply only to spatially uniform systems such as infinite nuclear matter

Consider a field theory with a Noether current, $J_{\mu}$, which is nearly conserved. That is, the divergence of the current can be written as

$$
\partial^{\mu} J_{\mu}=\lambda D
$$

where $D$ is a local operator and $\lambda$ is a parameter in the Lagrangian which can be made arbitrarily small. The theory has an exact symmetry in the limit $\lambda \rightarrow 0$. The symmetry is spontaneously broken if $\lim _{\lambda \rightarrow 0^{+}}\left\langle[Q, D]_{\mathrm{ET}}\right\rangle \neq 0$, where the subscript ET indicates equal time, $Q=\int \mathrm{d}^{3} x J_{0}$, and the brackets indicate a ground-state expectation value. The quantity $\lim _{\lambda \rightarrow 0^{+}}\left\langle[Q, D]_{\mathrm{ET}}\right\rangle$ is called the condensate, and is often thought of as the order parameter associated with the symmetry breaking [2] As is well known, spontaneous symmetry breaking (in the absence of a Higgs mechanism) implies the existence of massless Goldstone bosons 
[3]. Moreover, as shown by Gell-Mann, Oakes and Renner (GMOR) [4], if the explicit breaking parameter, $\lambda$, is nonzero then the square of the mass of the pseudo-Goldstone boson is proportional to $\lambda$.

Implicit in the usual derivation of the GMOR relation is the fact that the condensate is negative semi-definite. Thus, a condensate of zero is special. Of course, a vanishing condensate obviously occurs when the symmetry is not spontaneously broken. In the present Letter, however, we consider a different possibility: suppose the condensate vanishes, i.e.

$$
\lim _{\lambda \rightarrow 0}\left\langle[Q, D]_{\mathrm{ET}}\right\rangle=0,
$$

but that nevertheless the symmetry remains spontaneously broken. We will demonstrate here that this implies that the corresponding pseudo-Goldstone bosons must be anomalously light, i.e. the energy of the pseudo-Goldstone modes increases with $\lambda$ more slowly than $\lambda^{1 / 2}$. This means that

$$
\lim _{\lambda \rightarrow 0+} \frac{E_{\mathrm{PGB}}^{2}}{\lambda}=0
$$

(where $E_{\mathrm{PGB}}$ is the energy of the pseudo-Goldstone boson), rather than the usual case, in which this limit gives a nonzero value. In fact, many years ago it was suggested that the chiral condensate of QCD vanishes in the vacuum despite the existence of spontaneous chiral symmetry breaking [5]. In this scenario the square of the pion mass is proportional to the square of the current quark mass rather than to the current quark mass itself. It is believed that this scenario is quite implausible in the context of $\mathrm{QCD}$ since it violates a naturalness condition - it depends on the condensate vanishing "accidently" [6].

The principal new result in this Letter is that the fact that a vanishing condensate in the presence of spontaneous symmetry breaking implies that the pseudo-Goldstone modes are anomalously light holds more generally than for the vacuum. In particular, the result can be generalized to any spatially uniform ground state of the theory (medium) subject to a constraint (such as a spatially uniform density of some conserved current) so long as the operator which specifies the constraint commutes with $Q$. This generalized condition 
has an important possible application in nuclear physics. The underlying dynamics is, of course, QCD, and the current of interest is the axial vector current. The condensate is $\langle\bar{q} q\rangle$, and the role of $\lambda$ is played by the current quark mass $m_{q}$. Because this result holds only for spatially uniform systems, we consider the problem of infinite nuclear matter at nonzero baryon density; since the constraint must commute with the chiral charges (which carry isospin) we restrict our attention to isospin symmetric nuclear matter.

There is general agreement that as the baryon density of infinite nuclear matter increases, the chiral condensate decreases [1, 17 14. It is possible that at a sufficiently high density the condensate vanishes. This may be a signature of the chiral restoration phase transition. However, as recently observed in a model by Ericson [1], the fact the condensate vanishes does not necessarily imply that the symmetry is restored - the expectation value of other chiral symmetry breaking operators may be nonzero. The relevance of the present general result in this context is clear: if Ericson's scenario is correct, and the chiral condensate vanishes without chiral restoration, then the pseudo-Goldstone modes, i.e. the pions [15], must be anomalously light. Such anomalously light pions are what one expects in the vicinity of an S-wave pion condensation phase transition. The existence of anomalously light pions in this situation is a nontrivial dynamical consequence deduced from general principles. Of course, without solving QCD at finite densities one does not know whether Ericson's scenario occurs in the real world. However, this scenario is realized in simple models of S-wave pion condensation based on mean field treatment of the nonlinear $\sigma$ model. By construction, these models always have spontaneous symmetry breaking. At some density the condensate vanishes. Simultaneously, the pion mass becomes anomalously light — indeed, it goes to zero and pion condensation occurs. Note that in the context of nuclear matter it is not "unnatural" for the condensate to vanish without chiral restoration, since the vanishing condensate is associated with a phase transition.

Now we come to the core of this Letter. Let us consider the general case, in which the state of interest is the ground state of the theory subject to a spatially uniform constraint. In our initial discussion we will ignore issues associated with renormalization. As we will argue 
later, the renormalization effects do not affect our conclusions. The constraint is imposed by insisting that the expectation value of some local constraint operator, $C(x)$, in the state of interest is equal to some fixed value independent of $x$. Here we consider the case where the constraint operator commutes with the charge associated with the Noether current $J$, that is

$$
[Q, C(x)]_{\mathrm{ET}}=\left[\int \mathrm{d}^{3} y J_{0}(y), C(x)\right]_{\mathrm{ET}}=0
$$

Let us denote the ground state subject to the constraint by $|C\rangle$ (the vacuum is simply the special case for which $C=0$ ). Since, our constraint is spatially uniform, the constraint commutes with the three-momentum. We define

$$
G_{B}^{C}(\lambda) \equiv i\left\langle C\left|[Q, B(0)]_{\mathrm{ET}}\right| C\right\rangle_{\lambda}
$$

where $\mathrm{B}$ is an arbitrary local operator independent of $\lambda$, and the subscript $\lambda$ indicates a fixed value of $\lambda$ and a fixed constraint. We assume that the symmetry is spontaneously broken, which implies that there exists some operator $B$ for which $\lim _{\lambda \rightarrow 0^{+}} G_{B}^{C}(\lambda) \neq 0$.

The key issue in the analysis is the insertion of a complete set of intermediate states into Eq. (5). Since the medium is spatially uniform, these states can be labeled by their three-momentum relative to the state $|C\rangle$, and an additional label $j$. Now let us denote the energy of the the state relative to the state $|C\rangle$ as $E_{j}(\vec{p})$. Note $E_{j}$ and $\vec{p}$ are not the absolute energy and momentum of the state, but the difference of the energy and momentum of the state from the ground state. For example, if the state is a single quasiparticle state, $E_{j}(p)$ and $\vec{p}$ are the natural variables to describe the energy and momentum of the quasiparticle. Since the total energy and total momentum of the state labeled $j$ and of the ground state (subject to constraints) are both four-vectors, their difference is also a four-vector; hence $E_{j}$ and $\vec{p}$ form a four-vector and $E_{j}^{2}-p^{2}$ is a Lorentz scalar. Unity can be decomposed as

$$
1=\sum_{j} \int \frac{\mathrm{d}^{3} p}{(2 \pi)^{3} 2\left|E_{j}(p)\right|}|j, \vec{p}\rangle\langle j, \vec{p}|,
$$

where the factor $\left(2\left|E_{j}(\vec{p})\right|\right)^{-1}$ is included to ensure the Lorentz invariance of the measure, and the states have the standard covariant normalization $\langle j, \vec{k} \mid j, \vec{p}\rangle=2\left|E_{j}(p)\right| \delta(\vec{p}-\vec{k})$. Note 
the absolute value signs on the energy. Of course, if $|C\rangle$ is the vacuum, then $E_{j}$ must be positive. Of course, for $C \neq 0$ there are, in general, states lower in energy than $|C\rangle$. As we shall see, however, given the assumption that our constraint commutes with the charge, intermediate states with $E_{j}<0$ will not contribute to $G_{B}^{C}(\lambda)$.

Inserting Eq. (6) into Eq. (5) yields

$$
G_{B}^{C}(\lambda)=-\sum_{j} E_{j}^{-1} \operatorname{Im}\left(\left\langle C\left|J_{0}(0)\right| j, \vec{p}=0\right\rangle_{\lambda}\langle j, \vec{p}=0|B(0)| C\rangle_{\lambda}\right)
$$

where $E_{j}$ implicitly refers to the state with $\vec{p}=0$. Note that at this step we have dropped the absolute value sign. The reason is the condition in Eq. (田) that the charge in question commutes with the constraint, plus the fact that $|C\rangle$ is the minimum energy state subject to the constraint. Thus, the only states coupled to $|C\rangle$ by $Q$ are states with an energy greater than or equal to the energy of $|C\rangle$.

One can use Eq. (11) to relate matrix elements of $J_{0}$ in Eq. (7) to matrix elements of $D$ :

$$
\left\langle j, \vec{p}=0\left|J_{0}(0)\right| C\right\rangle_{\lambda}=-i \frac{\lambda}{E_{j}}\langle j, \vec{p}=0|D(0)| C\rangle_{\lambda} .
$$

Inserting this into Eq. ([]) gives

$$
G_{B}^{C}(\lambda)=-\sum_{j} \frac{\lambda}{E_{j}^{2}} \operatorname{Re}\left(\langle C|D(0)| j, \vec{p}=0\rangle_{\lambda}\langle j, \vec{p}=0|B(0)| C\rangle_{\lambda}\right) .
$$

An important special case is where the operator $B$ is itself $D$. In this case Eq. (9) becomes

$$
G_{D}^{C}(\lambda)=-\sum_{j} \frac{\lambda}{E_{j}^{2}}\left|\langle C|D(0)| j, \vec{p}=0\rangle_{\lambda}\right|^{2}
$$

The limit of $G_{D}^{C}(\lambda)$ as $\lambda \rightarrow 0^{+}$is, by definition, the condensate. To proceed we need to make the rather innocuous assumption that the matrix elements $\langle C|D(0)| j, \vec{p}=0\rangle_{\lambda}$ and $\langle C|B(0)| j, \vec{p}=0\rangle_{\lambda}$ do not diverge as $\lambda \rightarrow 0^{+}$. Given this assumption, the condensate can be written as

$$
\lim _{\lambda \rightarrow 0^{+}} G_{D}^{C}(\lambda)=-\sum_{j}\left|\langle C|D(0)| j, \vec{p}=0\rangle_{\lambda=0}\right|^{2} \lim _{\lambda \rightarrow 0^{+}} \frac{\lambda}{E_{j}^{2}} .
$$

Note that every term on the right-hand side of Eq. (11) is negative semi-definite. Thus, we observe that the condensate is negative semi-definite. This is a rather general corollary of 
our derivation, which extends the vacuum result to the constrained state $|C\rangle$. It is worth noting that this result depends in an essential way on the fact that our constraint commutes with the charges so that the only states connected have an energy greater than or equal to that of $|C\rangle$.

Furthermore, if the condensate is nonzero, there must exist at least one term in the sum which is nonzero. Given, the assumption that $\langle C|D(0)| j, \vec{p}=0\rangle_{\lambda=0}$ is finite, one concludes there must be at least one term in the summation for which $\lim _{\lambda \rightarrow 0^{+}} \frac{\lambda}{E_{j}^{2}} \neq 0$. Such a mode is a pseudo-Goldstone mode. As expected, the square of the energy of this state is proportional to the symmetry breaking parameter, $\lambda$. Equation (11) may be viewed as an in-medium generalization of the GMOR relation.

Now consider the situation in which the condensate is zero. Since each term in Eq. (11) is negative semi-definite, this implies that the limit of each term as $\lambda \rightarrow 0^{+}$must be zero. One solution is that the symmetry has been restored. Let us suppose, however, that the symmetry remains spontaneously broken. This means that there exists some operator $B$ for which $\lim _{\lambda \rightarrow 0^{+}} G_{B}^{C}(\lambda) \neq 0$, which implies that at least one term in Eq. (9) is nonzero. For both of these situations to be simultaneously true, there must exist some mode $j$ for which

$$
\begin{gathered}
\lim _{\lambda \rightarrow 0^{+}} \frac{\lambda}{E_{j}^{2}} \cos \left(\phi_{B D}^{j}\right)\left|\langle C|D(0)| j, \vec{p}=0\rangle_{\lambda}\right|\left|\langle j, \vec{p}=0|B(0)| C\rangle_{\lambda}\right|=a_{j} \neq 0 \\
\lim _{\lambda \rightarrow 0^{+}} \frac{\lambda}{E_{j}^{2}}\left|\langle C|D(0)| j, \vec{p}=0\rangle_{\lambda}\right|^{2}=0
\end{gathered}
$$

where $\cos \left(\phi_{B D}^{j}\right)$ is the relative phase between the matrix elements of $D$ and $B$. Since by hypothesis $\langle j, \vec{p}=0|B(0)| C\rangle_{\lambda=0}$ is not divergent, Eq. (12) can be satisfied only if

$$
\lim _{\lambda \rightarrow 0^{+}} \frac{\lambda}{E_{j}^{2}}\left|\langle C|D(0)| j, \vec{p}=0\rangle_{\lambda}\right|=\frac{a_{j}}{\cos \left(\phi_{B D}^{j}\right)\left|\langle j, \vec{p}=0|B(0)| C\rangle_{\lambda=0}\right|} .
$$

Equations (13) and (14) together imply $\lim _{\lambda \rightarrow 0^{+}}\left|\langle C|D(0)| j, \vec{p}=0\rangle_{\lambda}\right|=0$, which combined with Eq. (14) implies that $\lim _{\lambda \rightarrow 0^{+}} \frac{\lambda}{E_{j}^{2}} \rightarrow \infty$. This completes the demonstration that spontaneous symmetry breaking with a vanishing condensate implies the existence of an anomalously light mode - i.e. a mode for which 


$$
\lim _{\lambda \rightarrow 0^{+}} \frac{E_{j}^{2}}{\lambda}=0 .
$$

As mentioned previously, the preceding discussion ignored renormalization. It should be apparent, however, that renormalization effects will not alter the results. The operators $J_{\mu}, D$ and $B$ should be taken to be the appropriate renormalized operators. The only issue which needs to be addressed is the fact the operator product in Eq. (4) needs to be renormalized. Since we are ultimately treating these operator products via summation over intermediate states, as in Eq. (7), the renormalization of the composite operator is equivalent to the inclusion of ultraviolet subtraction constants in the summation. The key point in the present analysis, however, is that in the limit $\lambda \rightarrow 0$, the only intermediate states contributing to the sum are the pseudo-Goldstone modes. This is clear from Eq. (77-8) where the overall factor of $\lambda$ causes all terms to vanish in this limit except terms associated with modes whose mass goes to zero as $\lambda \rightarrow 0$. Thus, in this limit, there are no contributions from high mass states and hence no ultraviolet divergences. Since the result derived here is formally valid only in the limit $\lambda \rightarrow 0$, it is clear that it is not destroyed by renormalization.

Now, let us proceed to some applications of our general result. In the context of $S U(2) \times$ $S U(2)$ chiral symmetry in spatially uniform isoscalar nuclear matter, we have three partially conserved axial vector currents, $J_{5}^{a}$, the parameter $\lambda$ is the average up and down current quark mass, $m_{q}$, the condensate is $\lim _{m_{q} \rightarrow 0^{+}}\langle\bar{q} q\rangle$, and the (isospin degenerate) Goldstone modes are the pions: $\pi^{0}, \pi^{+}$and $\pi^{-}$. Thus, if $\langle\bar{q} q\rangle=0$ and chiral symmetry is not restored, the pion becomes anomalously light. As noted above, this is what one expects in the vicinity of an s-wave pion condensation transition.

We can also draw conclusions for isospin asymmetric nuclear matter, but only for the properties of the neutral pion, $\pi^{0}$. This is because the constraint operator, which in this case is the third isospin component of the vector charge, $J^{3}$, commutes only with the third isospin component of the axial vector charge, $Q_{5}^{3}$, and does not commute with $Q_{5}^{1}$ and $Q_{5}^{2}$. Hence we are only allowed to rotate about the third isospin direction, and the relevant symmetry is the $U(1) \times U(1)$ subgroup of the full chiral $S U(2) \times S U(2)$. Thus, the assumptions of our 
theorem are met for the case of the neutral pion, which becomes anomalously light if the condensate vanishes and the chiral $U(1) \times U(1)$ remains spontaneously broken.

Of course, it is an open question for infinite uniform nuclear mattter in QCD whether $\lim _{\lambda \rightarrow 0^{+}}\langle\bar{q} q\rangle$ ever goes to zero without chiral restoration. However, this scenario can be realized in simple models such as mean field treatments of the nonlinear $\sigma$ model. Such models have been used to explore the possibility of both $\pi$ and $K$ condensation 16 20]. Here, let us consider an $S U(2) \times S U(2)$ version of the model. The principal building block of the theory is the chiral field

$$
U=\exp \left(i \frac{\pi \cdot \tau}{f_{\pi}}\right),
$$

where $\pi$ is the pion field and $\tau$ represents the Pauli matrices. The commutator of $U$ with the axial vector charge is given by

$$
i\left[Q_{a}^{5}, U\right]=i f_{\pi} \partial_{\pi_{a}} U .
$$

In these models, $U$ is taken to be the only chirally active degree of freedom; all other degrees of freedom (such as baryons) commute with the axial vector charge. Thus, for these models the condensate, which we denote $\langle\bar{q} q\rangle$ in analogy to the equivalent quantity in $\mathrm{QCD}$, is given by

$$
\langle\bar{q} q\rangle=\frac{\left\langle f_{\pi}^{2} \partial_{\pi_{a}}^{2} \mathcal{L}^{\chi s b}\right\rangle}{m_{q}},
$$

where $\mathcal{L}^{x s b}$ is the chiral symmetry breaking part of the sigma model Lagrangian. It is therefore apparent that a vanishing condensate implies $\left\langle\partial_{\pi_{a}}^{2} \mathcal{L}^{\chi s b}\right\rangle=0$, which means that the pions have zero excitation energy. A zero pion energy is consistent with our result since it is anomalously light. In a simple mean field model [21] with heavy nondynamical nucleons (i.e. ignoring all terms second order in the baryon density) this occurs at the density of $m_{\pi}^{2} f_{\pi}^{2} / \sigma_{\pi N}$, where $\sigma_{\pi N}$ is the $\pi$-N sigma term. In the context of such models, this is taken to be the point at which s-wave pion condensation occurs. As stressed by Yabu, Myhrer and Kubodera [21], however, this result is highly model dependent and can easily be altered by the inclusion of effects second order in the density. 
We have yet to address an extremely important issue. Our general result was derived in the limit that $\lambda \rightarrow 0^{+}$. In applications to chiral symmetry in nuclear physics the parameter is some combination of quark masses and these are small but not zero. Clearly, if the masses are small enough the physics will be essentially unchanged from the zero mass limit. The important question is "How small is small enough?" Going back to the derivation the answer is clear. The result applies qualitatively, so long as the state $|C\rangle$ for a finite quark mass is essentially the same as the state in the massless quark limit (in the sense that the various matrix elements of $|C\rangle$ which enter the derivation are nearly the same as for the massless quark case). This restriction is very important. While we have discussed the case of s-wave pion condensation, we have not addressed the more interesting case of s-wave kaon condensation which is generally believed to be far more probable. Our general result, unfortunately, tells us nothing about this problem. The difficulty is that in the context of our derivation the strange quark mass cannot be regarded as small. The problem of interest is the transition at some critical density from ordinary nuclear matter (with zero net strangeness) to a condensed phase. However, in the limit $m_{s}, m_{u}, m_{d} \rightarrow 0$ ordinary nuclear matter is never the ground state of the theory subject to a constraint of fixed baryon density; $\mathrm{SU}(3)$ symmetric matter, with the strange quark density equal to $1 / 3$ of the baryon density is the ground state. Thus, the ordinary nuclear matter state for physical quark masses is qualitatively changed as one goes to the chiral limit and our general result tells us nothing. One might suppose that the problem can be circumvented by using the density of up and down quarks (rather than the baryon density) as the constraint. While this does prevent the ground state from changing qualitatively as one goes to the chiral limit, it still does not allow the application of our general result since the axial currents containing strange quarks (which are connected to the modes of interest for kaons) now do not commute with the constraint which violates a necessary condition for our theorem. The case of non-commuting constraints will be reported elsewhere [22].

We have demonstrated a very general result and shown its implications to descriptions of nuclear matter. We have not yet addressed one important issue, the role of correlations 
in $\langle\bar{q} q\rangle$. The essential physical point stressed by Ericson [1] is that the chiral condensate is an average quantity. In a reasonable description of nuclear matter there are very strong spatial correlations in the local value of $\langle\bar{q} q(x)\rangle$. In essence, one expects that near (and inside) nucleons the condensate is very different from its vacuum value while between nucleons the value of $\langle\bar{q} q(x)\rangle$ may not be much altered from the vacuum. Given this picture, it is reasonable to ask whether this average quantity should play any special role. It apparently represents the average of two very different kinds of physics and one might imagine that it could go to zero or even change signs without anything special (such as chiral restoration) occurring. For this to happen, it seems that all that must occur is that the positive contributions in the vicinity of nucleons has to become larger than the negative contributions between nucleons.

The formal derivation outlined in this letter does not specifically address the question of correlations. However, it does show that intuition based on the existence of strong correlations can be misleading. The chiral condensate — the averaged quantity — is special. For example, from very general considerations, one sees that it is negative semi-definite (to leading order in $m_{q}$ ). A vanishing chiral condensate, despite its average nature, has strong implications for the dynamics: implying either chiral restoration or anomalously light pions.

To summarize, we have shown that for the ground state of a system subject to a constraint which commutes a nearly conserved current, a vanishing condensate implies either that the symmetry is not spontaneously broken or that the pseudo-Goldstone modes are anomalously light. In the context of nuclear physics, this implies that if the chiral condensate vanishes for isoscalar nuclear matter at some density, then this density is at or near the critical density for either an S-wave pion condensation phase transition or a chiral restoration phase transition.

We acknowledge useful discussions with G. E. Brown, M. K. Banerjee and H. Forkel. This work has been supported by the NSF-Polish Academy of Science grant \#INT-9313988, NSF PYI grant \#PHY-9058487, DOE grant \#DE-FG02-40762, and partly by the Polish State Committee for Scientific Research grants 2.0204.91.01 and 2.0091.91.01, by the Maria Skłodowska-Curie grant PAA/NSF-94-158, and by the Alexander von Humboldt Foundation. 


\section{REFERENCES}

[1] T. Ericson, Phys. Lett. B321, 312 (1994).

[2] Note that our definition of the condensate has the symmetry limit $\lim _{\lambda \rightarrow 0^{+}}$built in. Thus, e.g. in the case of the chiral symmetry, our definition corresponds to the chirally leading piece of $\langle\bar{q} q\rangle$.

[3] J. Goldstone, Nuovo Cim. 19, 154 (1961).

[4] M. Gell-Mann, R. Oakes and B. Renner, Phys. Rev. 175, 2195 (1968).

[5] This scenario is part of a scheme called Neutral PCAC. A review of this approach may be found in M. D. Scadron, Rep. Prog. Phys. 44, 213 (1981).

[6] J. Gasser and H. Leutwyler, Phys. Rep. 87, 77 (1982).

[7] T. D. Cohen, R. J. Furnstahl, D. K. Griegel, Phys. Rev. C 45, 1881 (1992); Phys. Rev. Let. 67, 961 (1991).

[8] E. G. Drukarev and E. H. Levin, Nucl. Phys. A 51 679, (1990); A516, 715(E) (1990); Prog. Part. Nuc. Phys. 27, 77 (1991).

[9] M. Lutz, S. Klimt, W. Weise, Nucl. Phys. A 542, 521 (1992).

[10] L. S. Celenza, C. M. Shakin, W. Dong and X. Zhu, Phys. Rev. C 48, 159 (1993).

[11] M. Ericson, Phys. Lett. B301, 11 (1993).

[12] G. Chanfrey and M. Ericson, Nucl. Phys. A556, 427 (1993).

[13] M. C. Birse and J. A. McGovern, Phys. Lett B309, 234 (1993).

[14] M. C. Birse, University of Manchester preprint MC/TH 94/13.

[15] The concept of pions in nuclear medium is somewhat subtle. By pions, we mean excitations with the quantum numbers of the pion. These excitations may look very different than pions in the vacuum, e.g. they will involve particle-hole excitations. Neverthe- 
less, general statements of this Letter apply to these states without a reference to the dynamics.

[16] D. Montano, H. D. Politzer and M. B. Wise, Nucl. Phys. B375, 507 (1992).

[17] H. D. Politzer and M. B. Wise, Phys. Lett. B273, 156 (1991).

[18] D. B. Kaplan and A. E. Nelson, Phys. Lett. B175, 57 (1986); B192, 193 (1987).

[19] G. E. Brown, K. Kubodera, M. Rho, V. Thorsson, Phys. Lett. B291, 355 (1992).

[20] G. E. Brown, C.-H. Lee, M. Rho, V. Thorsson, Nucl. Phys. A567, 937 (1994).

[21] H. Yabu, F. Myhrer, K. Kubodera, Phys. Rev. D50, 1994.

[22] T. D. Cohen and W. Broniowski, to be published. 